\title{
Perception of risk and benefit in patient-centered communication and care
}

This article was published in the following Dove Press journal:

Patient Intelligence

26 May 2011

Number of times this article has been viewed

\section{Amin Hakim \\ Healthcare Consulting, Staten Island, NY, USA}

\begin{abstract}
There has been an increase in the adoption of patient-centered communication and accountable care that has generated greater interest in understanding patient perception of risk and benefit (PPRB). Patients find complex medical information hard to understand, resulting in inaccurate conclusions. Health behavior models describe the processes that individuals use to arrive at decisions concerning their own care. Studies have shown that their perception and decision making are associated with many factors such as age, gender, race, past experience, cost, and familiarity. Communication plays an important role in health literacy, and many adults are not proficient in the latter, regardless of their education. Clinicians have long provided educational materials but as our understanding of practitioner-patient communication and PPRB increased, so has the need for better ways to present medical advice and potential outcomes to the patient. Educational materials should be accessible, understandable, and actionable. They should have a reading level of grade 5 or 6 , and where possible include graphical representations. New print and multimedia tools incorporate easier to understand summaries of risks and benefits, but they often need additional improvements. Patients frequently have a great desire to share in decision making regarding their health, and may prefer to do so in a collaborative fashion with their health care providers. A shared decision will have the patient's input and promises better clinical outcomes as suggested by the literature; however, evidence from randomized controlled trials is scant. Additional studies should examine these and other types of outcomes. Patients tend to delegate decision making to clinicians in emergent or serious conditions. Practitioners need to have a positive communication style that engages patients in a shared decision making process and elicits the latter's preferences. Clinicians and patients often have divergent views on desired interventions and outcomes, and providers should be aware of their personal biases.
\end{abstract}

Keywords: perception, risk, benefit, preference, patient-centered, communication

\section{Introduction}

An understanding of patient's perceptions of risks and benefits (PPRB) of medical interventions is intuitively important in rendering care because it is associated with one's understanding of diagnosis and treatment, and consequently managing one's appointments, filling prescriptions, taking medications, and navigating both information resources and the care system. A body of knowledge regarding PPRB has accumulated over the past five decades as clinicians and health care organizations focus increasingly on patient-driven choices through shared decision making and informed consent.

The convergence of this focus and health care reform in the United States, England, and elsewhere is driving new approaches toward patient-centered accountable care with designs such as the chronic care model, medical home, and integrated care systems. 
While the details may differ among these models, they generally entail well-informed patients who self-manage their conditions towards better outcomes. In the US, physicians must demonstrate partnership with patients in developing care plans and goals and effective communication, particularly with vulnerable populations in order to receive recognition as patient-centered medical homes from the National Committee for Quality Assurance (NCQA).

Educating individuals and incorporating their preferences in treatment decisions are aspects of patient-centered care that hold promise for quality improvement, eg, in a large study by the Veterans Health Administration in the US, patientcentered care domains that correlated best with receipt of prevention guidelines were incorporating patient preferences in care and overall care coordination, ${ }^{1}$ and patients experienced better short-term recovery, emotional health, and fewer diagnostic tests and referrals if they had perceived patient-centeredness in provider communication (based on three aspects: exploring the illness experience, understanding the whole person, and shared decision making). ${ }^{2}$ This paper will review recent findings and additional work needed in the area of PPRB.

\section{Scope and methodology}

This article is a review of key aspects of PPRB, namely, its theoretical basis, influencing factors, shared decision making, decision aids, health literacy, clinician's role, and future research. Medline and Google searches used key words (perception, preference, risk, benefit,) to identify articles published in English particularly between 2000 and 2010. Additional terms were used, eg, patient-centered, communication, decision, and consent, and bibliographies of all publications were also searched. This review uses select reports to illustrate key factors and findings associated with PPRB as the literature review showed that many studies of PPRB focus on certain diseases, eg, cardiovascular conditions or specific drugs used in such diseases, and that significant methodological variations exist among them, with a small number being of high quality. References herein were selected because they demonstrate pertinent findings for a broad set of conditions and interventions, diverse types of patients and situations, various countries, and consistency or divergence in results. Data are quoted to emphasize comparisons where this is relevant.

\section{Basis of PPRB}

Why do similar patients under comparable circumstances perceive risk and benefit differently? How do they actually arrive at their conclusions and determine what actions they will take? Several theories regarding risk perception and health behavior are briefly reviewed herein as a backdrop to examples of PPRB in various situations.

The literature suggests that people's decisions are not rational and that as lessons learned from past experiences increase, so does one's preference to operate on the crudest gist, the fuzzy-processing preference, or "fuzzy trace theory". Giving patients precise information would be ineffective because people use simple bottom-line gist of information to make decisions instead of framing details, even when they can recall the latter precisely. During the decision making process, individuals apply their values, principles, knowledge, and reasoning. Health information is unclear to most patients, and poor recall is common and increases over time. Therefore erroneous judgment can result from information overload and/or processing interference, eg, when one tries to comprehend confusing details or numerical data presented simultaneously.

Lloyd ${ }^{4}$ reviewed the role of heuristics (decision making shortcuts developed from experience, eg, trial and error, that one uses to speed up problem solving and other cognitive functions), and noted how factors such as immediacy, controllability, novelty, and severity (catastrophic risk) may result in overestimation of harm. Optimism bias and cognitive avoidance (denial) account for people's underestimation of personal risk while viewing consequences as more hazardous to others. In the "health belief model" an individual's choices and "readiness to act" are based on four constructs: one's perceived susceptibility to disease, perceived severity of illness, perceived costs of health seeking behavior, and perceived benefits of action. The "cues to action" emerged as a concept whereby certain persuasive messages (advice from or illness in a partner, public campaigns) or interpersonal interaction activate a person's readiness and stimulate overt behavior such as the adoption of safer sex. ${ }^{5}$

One's relative weighing of pros and cons, the "decisional balance theory", is an important construct in the transtheoretical (TTM) model, which also encompasses self-efficacy, stages of change, and process of change. The stages of change are precontemplation or lack of intent for action, contemplation of change, preparation for action, action resulting in behavior change, maintenance to avoid relapse (recycling) during a period of 6 months to 5 years, and termination when one achieves $100 \%$ self-efficacy and $0 \%$ temptation. The model has been refined since its original description in 1977, and has received criticism regarding the efficacy of TTM-based interventions, which appears to be the case. ${ }^{6}$ 
But a majority of such interventions do not address the model's dimensions, and additional work is needed to understand the implications of this model in behavior modification. ${ }^{7}$ Other models include the social cognitive theory, and the theories of planned behavior, reasoned action, protection motivation, and self-efficacy. The self-efficacy concept emerged over 20 years ago and has been integrated formally or informally in several theories, eg, the health belief model. Sirur et $\mathrm{al}^{8}$ reviewed adherence to treatment in rehabilitation in the context of these models.

\section{Factors of PPRB}

Several reports are summarized in this section to highlight factors influencing PPRB. Researchers found that sickle-cell disease patient's willingness to accept mortality risk varied by the probability of death versus cure in allogenic stem cell transplantation; as much as $46 \%$ would accept $15 \%$ or greater risk of 30-day mortality. ${ }^{9}$ But $9 \%$ of them would accept the procedure only with $100 \%$ and $0 \%$ chances of cure and death, respectively, and would reject it at a 95\%-5\% split. Greek kidney transplantation candidates were unwilling to trade-off life years possibly because of strong religious beliefs and higher perception of risk in the gamble of transplantation. ${ }^{10}$ Additionally, $40.5 \%$ of them were unwilling to pay at all for transplantation while previously transplanted patients who probably experienced transient benefits were more likely to pay. Participation in religious activities correlated with greater risk perception and lower risk-seeking behaviors, eg, unprotected sex and violence, among American adolescents. ${ }^{11,12}$ Ethnicity was found to be an independent factor of greater risk perception among non-European patients than their European counterparts undergoing joint replacement in Canada, ${ }^{13}$ and could interfere with preventive care among Australian women of non-European ethnicity. ${ }^{14}$

Smoking is an area of great interest because of its significant impact on health and health care costs to individuals and the public, and is more challenging in adolescents because of their risk-taking behaviors. Research shows that risk-averse Japanese smokers who valued the benefits of cessation were less likely to relapse. ${ }^{15}$ Song et al ${ }^{16}$ investigated whether smoking-related perceptions predicted its initiation among American students in the 1st and 2nd years of high school (secondary education). The students believed that the chances of risks and social benefits (eg, "looking cool" or "feeling grown-up") were approximately $78 \%$ and $24.4 \%$, respectively. The lowest perceptions of risk and the highest perception of benefit were associated with approximately 3-fold likelihood of smoking initiation.
Tsui et a ${ }^{17}$ reported that the majority of their Canadian patients with acute coronary syndrome preferred treatment with percutaneous coronary intervention (PCI) to tissue plasminogen activator (tPA) with $78.5 \%, 88.1 \%$, and $95.4 \%$ choosing PCI based on information regarding mortality benefit, stroke risk, and net clinical benefit (NCB), respectively. When patients considered cost, $87.7 \%$ of them preferred PCI over tPA. A comparison of tPA with streptokinase (SK) in the same study showed that when tPA's higher stroke risk was presented to patients, $88 \%$ chose SK. But $100 \%$ and $66.7 \%$ chose tPA when they learned its mortality benefit and $\mathrm{NCB}$, respectively. Regarding cost, $53.9 \%$ and $72.5 \%$ of them chose tPA depending on whether they or the government paid for it, respectively. While the study has some limitations, it illustrates how patients change their choices depending on individual aspects of treatment. They demonstrated risk aversion regarding stroke even if alternative treatment offered higher survival rates. Similar to other studies, $78.1 \%$ of them would defer the selection to their providers even if their preference for quality of life may be discordant with physicians' NCBbased preference. As the authors noted, patient choice is at risk of being manipulated especially when he or she finds an intervention alluring or easier to understand.

Familiarity with an intervention and the desire for a particular outcome can lead to different choices depending on the patient's age. Young Canadian children, particularly those between 6 and 9 years, preferred casting to pin-fixation of a hypothetical femoral fracture, apparently because of familiarity with the former. ${ }^{18}$ Parents and older kids were more likely to choose the pin-fixation, opting for the mobility it offered. Older individuals may be more attuned to the pros and cons of medical procedures as suggested by the decisional balance theory. Like other reports, the study demonstrated that children as young as 6 years of age can choose an option after considering its "good and bad" characteristics. In another study, parents preferred that children with occult bacteremia have transient painful experiences like venipuncture, intravenous treatment, and hospitalization rather than rare risks of severe outcomes like death and complicated meningitis. ${ }^{19}$ While the methodologies for assessing patient preferences differ among the studies and they sometimes have contradictory results regarding risk perception and tolerance, they show that parents have a high degree of risk aversion concerning their children.

Risk aversion was the primary reason $(59.8 \%)$ among Greek survey respondents who declined vaccination against pandemic H1N1 influenza, citing concern about its safety. ${ }^{20}$ Other reasons for rejecting the vaccine included perceptions 
about its efficacy and low severity of illness. Women were more likely than men to decline vaccination $(67.6 \%$ versus $54.4 \%$ ) and were more resolute about their choice with $51.8 \%$ indicating "definitely not". Past influenza immunization was associated with higher acceptance. Interestingly, over half of the respondents and households with chronic conditions, a target immunization group, would decline the vaccine, and there was no association between knowledge of the disease and foregoing vaccination. Vaccine rejection increased significantly over 2 months from $32.3 \%$ in week $35 \%$ to $45.8 \%$ in week 44 . One wonders whether the survey participants gave more weight to information they received from the media than through clinical channels.

It is understandable that asymptomatic people are less likely to accept risk than those who are symptomatic. Total cure by medication tallied the highest maximum acceptable risk of mortality (MARM) scores among British rheumatoid arthritis (RA) and ankylosing spondylitis (AS) patients, while relief of stiffness, return to normal functioning, and relief of pain scored lower rates. ${ }^{21}$ The MARM results correlated with worse health status, and were inversely related to the duration of disease. RA patients, who were more symptomatic and likely had more daily-living-activity disruptions than their SA counterparts, were willing to accept higher risk. These findings were similar to previous results, eg, among American and Canadian RA patients whose mortality risk acceptance correlated with duration of illness, and with better prior health status, lower income, and marital status. ${ }^{22}$

A study in Crohn's disease showed that preferences of asymptomatic Canadian patients for one of five postoperative treatments varied widely, and no clinical or demographic factors could predict them because individuals assessed the risks and benefits of each option differently based on their needs and lifestyles. ${ }^{23}$ Their rationale was based on the type and severity of side effects and risk of recurrence rather than the likelihood of side effects or the complexity of a particular regimen. There was an inverse relationship between cost and choice such that $1 / 3$ of patients would reverse their initial preference because of cost. Interestingly, a small but significant minority would take a medication if it were no better than no therapy or less effective than another one.

Almost 3/4 of American patients who were surveyed regarding one of four non-life-threatening conditions would prefer nondrug therapies to medications regardless of efficacy. ${ }^{24}$ Three hypothetical options for each condition were offered to them: a "safer" drug with 50\% effectiveness, a "riskier" one with 100\% effectiveness, and a home remedy. White patients were twice as likely as African Americans to choose the safer drug, and college education was associated with a similar trend. Risk aversion and willingness to accept current discomfort were stronger than demographic variables in predicting preference for home remedies, and that choice was strongest for hypertension versus sore throat, arthritis, or gastroenteritis. A survey of study-abroad American students showed low perception of travel-related health risks, which ranked lower than contaminated food/water, psychological distress, excessive sun exposure, assault, and motor vehicle accident. ${ }^{25}$ Among health problems, infectious diseases were more prominent $(70 \%)$ than vector and airborne infections perhaps because of students' familiarity with them.

Medication adherence is an important aspect of treatment for many patients, and the first step is filling prescriptions (primary compliance). A survey of American patients found that cost was not the main reason for primary noncompliance; instead, the perception of lack of efficacy and concern about side effects ranked highest, followed by cost and lack of symptoms. ${ }^{26}$ Among Italian patients with asthma, 41.8\% were uncertain whether therapy was necessary, $28.2 \%$ were fearful of adverse effects, and $15.9 \%$ did not consider benefit greater than risk. ${ }^{27}$ Depression and anxiety, common among patients with chronic disease, were associated with inadequate knowledge about asthma, inability to identify worsening signs, denial of illness limitations, and/or concern about medication side effects.

The PPRB literature shows the complexity and interplay of several factors such as age, race, gender, acuity of illness, certainty and timeline of outcomes, cost, education, experience, health literacy, perception of medical technology, community norms, benefit to family or society, and other factors. Studies also demonstrate how common misperception of pros and cons is among patients, and how their views and actions are influenced by the amount and presentation of information they receive. Decision making dialog is essential to informed choices.

\section{Shared decision making (SDM)}

Patients prefer to participate in treatment decisions, largely in a shared manner with their health care provider, and delegate to him/her a greater decision making role in more serious illnesses. ${ }^{17,28-30}$ Several factors are associated with these preferences, eg, gender, age, ethnicity, and culture. ${ }^{29,30}$ There has been a growing interest in SDM, particularly with the focus on patient-centered care, since Engel's writings 30-35 years ago regarding a new biopsychological model in clinical practice. ${ }^{31,32}$ Emanuel and Emanuel ${ }^{33}$ outlined four models of physician-patient relationship: paternalistic, 
informative, interpretive, and deliberative, and suggested a fifth model, instrumental. They recognized that each model is justified under certain circumstances, eg, the guardian physician role (paternalistic) in emergencies; outlined each model's drawbacks; and recommended the deliberative one, ie, a collaborative decision making process.

Simply put, SDM means patient involvement and participation in treatment decisions. A review of 418 articles published as of 2003 noted that there were 31 concepts and no common definition for SDM. ${ }^{34}$ The words "patient values/ preferences" and "options" appeared in only $67.1 \%$ and $50.9 \%$, respectively, of 161 articles that actually included conceptual definitions of SDM. Elwyn and Edwards (recommended reading) ${ }^{35}$ cited Charles et al's ${ }^{36}$ description of SDM as 'involvement of both the patient and the doctor, a sharing of information by both parties, both parties taking steps to build a consensus about the preferred treatment, and reaching an agreement about which treatment to implement'. The model has undergone refinements and evaluation, eg, flexibility toward diverse patient preferences, awareness of cultural differences, implications for public policy, and application in clinical settings. ${ }^{37-41}$

Despite a growing interest in SDM, it is challenging to apply it in clinical practice. Légaré et $\mathrm{al}^{42}$ observed that, not surprisingly, health care provider motivation is a key driver in implementing SDM. Practitioners, largely physicians, cite several implementation barriers particularly time constraints, perception of its (im)practicality and/or rigidity, and nonagreement with one or more aspects of SDM such as applicability to one's patients and clinical scenarios (eg, acute self-limited versus chronic diseases.) Other factors were lack of familiarity with SDM, misperception of patient preferences, and lack of resources and motivation. A recent Cochrane review of factors leading to successful implementation of SDM reported that of 6764 publications, five studies - all randomized, controlled studies in the ambulatory setting - met the review criteria, but only two studies showed a significant effect on SDM adoption. ${ }^{43}$

One can gain good insights into physicians' attitudes regarding patient engagement from Ampt et al's observations about their behavior to influence patient's lifestyle risk factors. ${ }^{44}$ While clinicians believed that educating patients was part of their professional responsibility, there was significant variability both within and between the practitioners in engagement and motivation of patients to change their lifestyles. Some of that variability is inherent to physicians' interest in certain medical conditions and vision of their role relative to other types of health care providers, but a significant part was also due to misperception of patient's motivation, reaction to severity of his/her risk factors, cost sensitivity, access to referral services, and uncertainty about benefit from support services. Other factors were practice demands and patterns, and lack of or limited training in motivational interviewing.

\section{Decision making aids and communication}

Low perception of risk, gaps in knowledge and nonadherence to prevention and therapy recommendations obviously pose a health risk to patients, and compel us to communicate better with them through a benefit-harm dialog. In a classic example of information with limited readability, only $50 \%$ of 104 asthma educational leaflets were rated below secondary education (high school). ${ }^{45}$ Furthermore, 11 (20\%) of 90 leaflets contained inaccurate or misleading statements that were inconsistent with the British Thoracic Society's recommendations at the time. More than one decade later, the average readability level of 45 leaflets was 12 th grade in one report, ${ }^{46}$ while all of 31 samples were at grade 6 or higher (a single pamphlet at grade 6; five of them contained major inaccuracies), ie, above the recommended grade 5 to 6 , in another study. ${ }^{47}$ Another challenge is how often patients seek medical information from nonclinical sources, eg, the main source (85\%) of travel health information for student travelers was youth-oriented travel guidebooks. ${ }^{25}$ Other sources included friends and family $(75 \%)$ and commercial internet sites (12\%).

The content, format, and quality of communication between patients and physicians greatly influence the assimilation of exchanged knowledge. Barriers such as cultural disparities and language have a significant impact on patient perceptions. For example, language concordance between physicians and foreign-born Latino patients rather than the latter's English proficiency resulted in less confusion and frustration and higher ratings of overall quality of care. ${ }^{48}$ Satisfaction with physicians and medical care correlated independently with communication, patient-centered decision making, and interpersonal style among African American, English- and Spanish-speaking Latino, and nonLatino white patients in California. ${ }^{49}$ Perceptions of lack of clarity, explanation of test results, compassionate/respectful health care provider, and disrespectful staff differed by race/ ethnicity and language, suggesting that certain aspects of interpersonal communication are more important to some patients than others.

How benefit-harm information is presented to the patient influences his/her decision. For example, Misselbrook and 
Armstrong ${ }^{50}$ demonstrated that the likelihood of accepting treatment for mild hypertension dropped from $92 \%$ to $75 \%$, $68 \%$, and $44 \%$ if risks were explained to patients in terms of relative risk reduction (RRR), absolute risk reduction model (ARR), number needed to treat (NNT), and personal probability of benefit, respectively, and concluded that it was neither clear how decision making can be fully shared nor what should constitute informed consent in that context. Other authors both advocated and debated the use of ARR rather than RRR (which suggests larger effects), natural frequencies, NNT, event postponement, numerical or graphical representation, and other methods to frame potential benefits and risks for the patient. ${ }^{51-54}$ Although NNT provides absolute risk framing, it is one of the methods patients comprehend the least. ${ }^{50,55}$ Paling ${ }^{54}$ advised providing estimated numbers and avoiding purely descriptive terms to explain risk, while Ryena $^{3}$ called efforts to increase the precision of information given to patients misguided and recommended a focus on qualitative relations among numbers. She noted that in order to achieve understanding by the recipient, benefit-harm information must appeal to gist-based intuition - a distillation of meaning from past similar events - instead of verbatim-based analysis. This is accomplished by removing all or some of the precise details, such as numbers, and replacing them with vague and imprecise descriptive words, eg, low, high, and some, which preserve the bottom-line meaning. The author believes that physicians should be flexible and accommodate patients at both ends of the spectrum, from preference for specific details to the odds in simple terms.

Several types of graphical decision aids (DAs), eg, a crowd chart, are available to help patients understand the meaning of complex numerical data. ${ }^{54,56,57}$ The use of both numerical and graphical representations may result in better understanding of risk reduction..$^{58}$ While graphic DAs are easier to comprehend for many people, ${ }^{56,59}$ not all of them are necessarily inspiring or understandable. Goldman et $\mathrm{al}^{57}$ reported that focus groups in New England, USA, deemed the crowd chart confusing and uninteresting while a bar graph representing risk-adjusted age was more impressive.

Digital DAs have also been studied, eg, computerized graphical-numerical framing, reduced decisional conflict immediately but not at 3 months among candidates for antithrombotic therapy, and, as in several other studies of decision aids, knowledge was not improved..$^{60}$ Computeranimated characters simulating face-to-face conversation with patients (embodied conversational agents) seem to be particularly useful among patients with low health literacy levels. ${ }^{61}$ Graphic DAs too can be associated with higher or lower perception of risk, eg, preference to avoid warfarin among patients who received digital and nondigital DAs. ${ }^{60,62}$ In contrast, parents who received information about pediatric pain control in graphic rather than text or table format perceived risks to be lower and benefits to be higher. ${ }^{59}$ As we learn more about the use of DAs, multimedia, or otherwise, it becomes evident that their design may unintentionally influence PPRB, eg, gender and race of actors portraying patients. ${ }^{63}$

Patient (and public) education should address both the benefits and harms of interventions and behaviors, because people's decisions are a balance of their perceptions of both aspects. For example, Song et al noted that efforts aimed at smoking cessation and abstention should not focus on long-term risks as they often do, and should aim to emphasize short-term social consequences and refute perceived benefits. ${ }^{16}$

Do decisional aids work? The answer is "it depends". A Cochrane review ${ }^{64}$ (recommended reading) showed that they improve patient's knowledge, accuracy of outcome perception, and active involvement in decision making. There is an impact on few clinical outcomes, use of elective surgery, hormone replacement therapy, and prostate cancer screening. Evidence is largely lacking for a positive effect in other conditions, eg, depression; anxiety; angina; medication adherence; mental health; or social functioning; and urinary, menstrual, and menopausal symptoms. Impact on health care costs was variable. Cochrane review concluded that studies were underpowered, subject to swings from under- and overutilization, and perhaps should focus on asking whether patients experience the outcomes they prefer even if they are not the clinically "sound" ones.

Once a decision is reached, the patient should receive a plan of action as a reminder of what was agreed upon with the physician. More than three decades ago, Ellis et al reported that patients' recall of diagnosis, general advice, and drug treatment, but not prognosis or follow-up plan, was better if they had received a summary form at discharge from hospital. ${ }^{65}$ The provision of a similar clinical summary to patients, and collaborative informed decision making are criteria in NCQA's recognition program for patient-centered medical home.

\section{Health literacy}

A discussion of communication, patient education, and SDM has to address health literacy (HL), which is defined as the degree to which an individual has the capacity to obtain, communicate, process, and understand basic health information 
and services in order to make appropriate health decisions. ${ }^{66}$ The 2003 National Assessment of Adult Literacy included the first large-scale evaluation of HL in the US (or elsewhere) ${ }^{67}$ $\mathrm{HL}$ results were categorized as proficient (12\%), intermediate $(52 \%)$, basic $(22 \%)$, and below basic (14\%.) Most (88\%) respondents were below the proficient level, ie, they lacked the ability to synthesize information from complex documents. Data from HL assessment in other countries are difficult to compare with those from the US because of methodological differences, eg, age of respondents and score ranges for each HL level. Nonetheless the results generally show that the majority of adults do not have proficiency levels of HL, and that a significant segment of them have very low HL. In Australia, where five levels of HL are utilized, $6 \%$ of adults have levels 4 and 5 (results for these levels were combined), and $19 \%$ are at level $1 .{ }^{68}$

Signs of possible low HL include lack of follow-through on tests and referrals, medication nonadherence, frequently missed appointments, prevarication about compliance, inability to recite drug names and purpose, avoidance of clarification questions, and medical errors. The elderly, language barriers, chronic disease, and minority groups are additional red flags. Several techniques and DAs are recommended to address low HL, and the underlying theme is accessible, understandable, and actionable information. Wolf et al ${ }^{69}$ reported that patients with lower HL were more likely to be embarrassed - between $19 \%$ and $48 \%$ of those at grade 6 or less - to tell the providers (and even their family and friends) about their reading inabilities, another barrier to effective SDM and successful clinical management. Encouragingly, most patients with low or marginal literacy deemed it helpful for providers to know that they may not understand instructions or not be able to read medication labels. HL screening tools, some in more than one language, are mainly used in research because they need further validation or are time consuming.

National assessments have demonstrated that HL levels, and consequently patients' decisions and actions, are strongly associated with their level of education. However, anyone regardless of education and experience - even medical personnel - can be challenged to obtain and understand medical information, ie, have low level of HL. In the US, proficient and below-basic HL levels were associated with education higher than grade 9 and lower than grade 6, respectively. Other factors include age, employment, income, parental education, occupation, trust in or skepticism of information, stress, language barriers, health status, and alcohol consumption. ${ }^{67,68,70}$ Individuals with special needs such as developmental, psychiatric, or mental disorders pose even greater challenges.

\section{Clinician's role}

Physician's misapplication of risk-reduction framing and bias can result in suboptimal recommendations. ${ }^{71}$ Clinicians are susceptible to factors such as life expectancy and therapeutic contraindications that may alter their clinical decisions even when they know patient preferences. ${ }^{72,73}$ Although many physicians believe that they should inform and educate their patients well, they vary in putting that concept into practice. ${ }^{44}$ Those who espouse patient-centered care exhibit better communication skills; however, they tend to do so more with patients they regard as adherent, involved, and good communicators. ${ }^{74}$ The association between communication and desirable outcomes, eg, patient satisfaction and compliance, is not limited to positive verbal cues like empathy, reassurance, patient-centered questioning, positive reinforcement, and courtesy, but also nonverbal ones such as head nodding, forward lean, and direct body orientation. ${ }^{75}$ Physicians may display more respect toward older or familiar patients, and their patients can accurately perceive lesser respect. ${ }^{76}$ Race may $^{76}$ or may not ${ }^{74}$ be associated with difficult communication. Significantly, patient-centered communication builds trust in the provider, and patients are less likely to seek health information elsewhere, eg, the Internet. ${ }^{77}$

Clinicians often have a different perspective from their patients', and should work toward better understanding of their preferences, eg, quality of life over clinical benefit. ${ }^{17,78-80}$ Clinicians should be attuned to how much of an active role patients want to play in decision making, and should not ask patients whether they understand their instructions or not, because patients may feel pressured to agree out of the desire to hide low literacy, or to avoid disappointing or disagreeing with the providers. They should instead utilize the teach-back method where patients recite back the information they grasped then providers fill in knowledge gaps and correct misunderstandings.

\section{Future research}

We need to determine the best ways to help patients make decisions favorable to their health outcomes while incorporating their preferences. Studies should be high quality, randomized controlled trials with sufficient power to evaluate different approaches to patient education in diverse populations, different countries, and varied health care settings. Research should be conducted in real clinical situations rather than hypothetical scenarios, and should include HL 
and multiple morbidities as confounding factors. Although we learned a great deal about patient-physician interaction, we need to further elucidate the patterns and effectiveness of communication with patients that promote SDM and desired outcomes. Researchers may examine how to choose the optimal decision aid formats appropriate for particular types of patients, eg, those with specific cultural or religious beliefs, certain diseases, or low HL; how to incorporate them in an overall strategy of patient-centered care; and when to modify ethically the scope of SDM.

DAs improve accuracy of PPRB, knowledge, and patient participation in SDM. There is an opportunity to enhance their impact on health and cost outcomes, and to evaluate new delivery methods, eg, integrating DAs with tools like personal health records (PHR) and patient-centered websites such as PatientsLikeMe, and offering customized health information based on not only one's demographic and clinical data but also personal preferences. Accessible internet-based offerings pose challenges limiting their widespread adoption; they require high levels of literacy and navigation skills. Therefore improvements in these two areas would provide patients with more accessible, understandable, and actionable information. We need to understand the role and effectiveness of new support tools for adherence and self-management, eg, mobile phone medication reminders, which are available as standalone or as a component of other products like PHR. It is necessary to conduct longitudinal studies of changes over time in PPRB, eg, treatment after relapse or adverse event, end-of-life choices, and of the effectiveness of different tools in achieving long-term compliance. Given the incremental cost of DAs in the face of limited resources, and the tendency of regulatory and accreditation agencies to add new requirements in that area, a comparative examination of their cost effectiveness is highly desirable.

\section{Conclusion}

Patients make suboptimal health decisions because they disproportionately value benefits and risks of medical interventions based on their perception of gain and loss, and poorly understood clinical information. Several factors influence patient's understanding of physician recommendations and their perception of the latter's clinical, monetary, and psychosocial harms and benefits. Patients expect clinicians to make the diagnosis, determine the treatment, and outline the pros and cons (problem solving), but most of them want to participate in decision making. Effective positive communication and shared decision making are important building blocks of patient-centered accountable care, and are important steps in reaching informed consent and better clinical outcomes. Communication should account for literacy limitations, avoid technical terms, and simplify complex risk-benefit data. Patient preferences for details versus descriptive bottomline terms, and for clinical versus nonclinical outcomes are important aspects in the risk-benefit dialog. There are many decision aids and other tools available to increase, at least transiently, patient knowledge and help them with near-future decisions. Attention to the frequency and role of health literacy is increasing. High quality studies of PPRB and SDM would help us identify better means to educate patients, and to assist them in reaching optimal results. Patient knowledge of his/her condition and management are improved at least in the short-term by incorporating postvisit summaries. Repetitive education and techniques borrowed from the advertising world may enhance patient perception, stall risk-taking behavior, and improve long-term adherence to prevention and healthy living recommendations.

\section{Acknowledgment}

The author thanks Ms Julia Hakim for her valuable assistance in the research and editing of this manuscript.

\section{Disclosure}

The author reports no conflicts of interest in this work.

\section{References}

1. Flach SD, McCoy KD, Vaughn TE, Ward MM, Bootsmiller BJ, Doebbeling BN. Does patient-centered care improve provision of preventive services? J Gen Intern Med. 2004;19(10):1019-1026.

2. Stewart M, Brown JB, Donner A, et al. The impact of patient-centered care on outcomes. J Fam Pract. 2000;49(9):796-804.

3. Reyna VF. A theory of medical decision making and health: fuzzy trace theory. Med Decis Making. 2008;28(6):850-865.

4. Lloyd AJ. Communicating and understanding risk. The extent of patients' understanding of the risk of treatments. Qual Health Care. 2001;10(Suppl 1):i14-i18.

5. Mattson M. Toward a reconceptualization of communication cues to action in the Health Belief Model: HIV test counseling. Commun Monogr. 1999;66(3):240-265.

6. Noar SM, Benac CN, Harris MS. Does tailoring matter? Metaanalytic review of tailored print health behavior change interventions. Psychological Bulletin. 2007;133(4):673-693.

7. Hutchison AJ, Breckon JD, Johnston LH. Physical activity behavior change interventions based on the transtheoretical model: a systematic review. Health Educ Behav. 2009;36(5):829-845.

8. Sirur R, Richardson J, Wishart L, Hanna S. The role of theory in increasing adherence to prescribed practice. Physiother Can. 2009; 61(2):68-77.

9. Van Besien K, Koshy M, Anderson-Shaw L, et al. Allogenic stem cell transplantation for sickle cell disease. A study of patients' decisions. Bone Marrow Transplant. 2001;28(6):545-549.

10. Kontodimopoulos N, Niakas D. Overcoming inherent problems of preference-based techniques for measuring health benefits: an empirical study in the context of kidney transplantation. BMC Health Serv Res. 2006;6:3. 
11. Miller L, Gur M. Religiousness and sexual responsibility in adolescent girls. J Adolesc Health. 2002;31(5):401-406.

12. Nonnemaker JM, McNeely CA, Blum RW. Public and private domains of religiosity and adolescent health risk behaviors: evidence from the National Longitudinal Study of Adolescent Health. Soc Sci Med. 2003; 57(11):2049-2054.

13. Gandhi R, Razak F, Davey JR, Mahomed NN. Ethnicity and patient's perception of risk in joint replacement surgery. J Rheumatol. 2008; 35(8):1664-1667.

14. Kwok C, Sullivan G, Cant R. The role of culture in breast health practices among Chinese-Australian women. Patient Educ Couns. 2006; 64(1-3):268-276.

15. Goto R, Takahashi Y, Nishimura S, Ida T. A cohort study to examine whether time and risk preference is related to smoking cessation success. Addiction. 2009;104(6):1018-1024.

16. Song AV, Morrell HE, Cornell JL, et al. Perceptions of smoking-related risks and benefits as predictors of adolescent smoking initiation. $\mathrm{Am} J$ Public Health. 2009;99(3):487-492.

17. Tsui W, Pierre K, Massel D. Patient reperfusion preferences in acute myocardial infarction: mortality versus stroke, benefits versus costs, high technology versus drugs. Can J Cardiol. 2005;21(5): 423-431.

18. Burke TM, Abramovitch R, Zlotkin S. Children's understanding of the risks and benefits associated with research. J Med Ethics. 2005; 31(12):715-720.

19. Bennett JE, Sumner W 2nd, Downs SM, Jaffe DM. Parents' utilities for outcomes of occult bacteremia. Arch Pediatr Adolesc Med. 2000; 154(1):43-48.

20. Sypsa V, Livanios T, Psichogiou M, et al. Public perceptions in relation to intention to receive pandemic influenza vaccination in a random population sample: evidence from a cross-sectional telephone survey. Euro Surveill. 2009;14(49):19437.

21. O'Brien BJ, Elswood J, Calin A. Willingness to accept risk in the treatment of rheumatic disease. J Epidemiol Community Health. 1990; 44(3):249-252.

22. Thompson MS. Willingness to pay and accept risks to cure chronic disease. Am J Public Health. 1986;76(4):392-396.

23. Kennedy ED, To T, Steinhart AH, Detsky A, Llewellyn-Thomas HA, McLeod RS. Do patients consider postoperative maintenance therapy for Crohn's disease worthwhile? Inflamm Bowel Dis. 2008;14(2) 224-235.

24. Povar GJ, Mantell M, Morris LA. Patients' therapeutic preferences in an ambulatory care setting. J Public Health. 1984;74(12):1395-1397.

25. Hartjes LB, Baumann LC, Henriques JB. Travel health risk perceptions and prevention behaviors of US study abroad students. $J$ Travel Med. 2009; 16(5):338-343.

26. Hakim A. E-prescribing and primary noncompliance: physician and patient experience. Prof Case Manag. 2010;15(2):62-67.

27. Baiardini I, Braido F, Giardini A, et al. Adherence to treatment: assessment of an unmet need in asthma. $J$ Investig Allergol Clin Immunol. 2006;16(4):218-223.

28. Deber RB, Kraetschmer N, Irvine J. What role do patients wish to play in treatment decision making? Arch Intern Med. 1996;156(13): 1414-1420.

29. Singh JA, Sloan JA, Atherton PJ, et al. Preferred roles in treatment decision making among patients with cancer: a pooled analysis of studies using the Control Preferences Scale. Am J Manag Care. 2010;16(9): 688-696.

30. Patel SR, Bakken S. Preferences for participation in decision making among ethnically diverse patients with anxiety and depression. Community Ment Health J. 2010;46(5):466-473.

31. Engel GL. The need for a new medical model: a challenge for biomedicine. Science. 1977;196(4286):129-136.

32. Engel GL. The clinical application of the biopsychosocial model. Am J Psychiatry. 1980;137(5):535-544.

33. Emanuel EJ, Emanuel LL. Four models of the physician-patient relationship. JAMA. 1992;267(16):2221-2224.
34. Makoul G, Clayman ML. An integrative model of shared decision making in medical encounters. Patient Educ Couns. 2006;60(3):301-312.

35. Elwyn G, Edwards A, editors. Shared decision-making in health care: achieving evidence-based patient choice. 2nd ed. New York: Oxford University Press; 2009.

36. Charles C, Gafni A, Whelan T. Shared decision-making in the medical encounter: what does it mean? (or it takes at least two to tango). Soc Sci Med. 1997;44(5):681-692.

37. Charles C, Gafni A, Whelan T. Decision-making in the physician-patient encounter: revisiting the shared treatment decision-making model. Soc Sci Med. 1999;49(5):651-661.

38. Charles C, Gafni A, Whelan T, O'Brien MA. Cultural influences on the physician-patient encounter: the case of shared treatment decisionmaking. Patient Educ Couns. 2006;63(3):262-267.

39. Murray E, Charles C, Gafni A. Shared decision-making in primary care: tailoring the Charles et al model to fit the context of general practice. Patient Educ Couns. 2006;62(2):205-211.

40. Elit L, Charles C, Gold I, et al. Women's perceptions about treatment decision making for ovarian cancer. Gynecol Oncol. 2003;88(2):89-95.

41. Dolan JG. Shared decision-making - transferring research into practice: the Analytic Hierarchy Process (AHP). Patient Educ Couns. 2008;73(3): $418-425$.

42. Légaré F, Ratté S, Gravel K, Graham ID. Barriers and facilitators to implementing shared decision-making in clinical practice: update of a systematic review of health professionals' perceptions. Patient Educ Couns. 2008;73(3):526-535.

43. Légaré F, Ratté S, Stacey D, et al. Interventions for improving the adoption of shared decision making by healthcare professionals. Cochrane Database Syst Rev. 2010;12(5):CD006732.

44. Ampt AJ, Amoroso C, Harris MF, McKenzie SH, Rose VK, Taggart JR. Attitudes, norms and controls influencing lifestyle risk factor management in general practice. BMC Fam Pract. 2009;10:59.

45. Smith H, Gooding S, Brown R, Frew A. Evaluation of readability and accuracy of information leaflets in general practice for patients with asthma. BMJ. 1998;317(7153):264-265.

46. Cronin M, O'Hanlon S, O'Connor M. Readability level of patient information leaflets for older people. Ir J Med Sci. 2011;180(1):139-142.

47. Nicholls S, Hankins M, Hooley C, Smith H. A survey of the quality and accuracy of information leaflets about skin cancer and sun-protective behaviour available from UK general practices and community pharmacies. J Eur Acad Dermatol Venereol. 2009;23(5):566-569.

48. González HM, Vega WA, Tarraf W. Health care quality perceptions among foreign-born Latinos and the importance of speaking the same language. J Am Board Fam Med. 2010;23(6):745-752.

49. Nápoles AM, Gregorich SE, Santoyo-Olsson J, O’Brien H, Stewart AL. Interpersonal processes of care and patient satisfaction: do associations differ by race, ethnicity, and language? Health Serv Res. 2009;44(4): $1326-1344$.

50. Misselbrook D, Armstrong D. Patients' responses to risk information about the benefits of treating hypertension. Br J Gen Pract. 2001; 51(465):276-279.

51. Neeskens P. Evidence, risk and the patient. Aust Prescr. 2007;30(2): $47-50$.

52. Schwartz PH, Meslin EM. The ethics of information: absolute risk reduction and patient understanding of screening. J Gen Intern Med. 2008;23(6):867-870.

53. Halvorsen PA, Selmer R, Kristiansen IS. Different ways to describe the benefits of risk-reducing treatments: a randomized trial. Ann Intern Med. 2007;146(12):848-856.

54. Paling J. Strategies to help patients understand risks. BMJ. 2003; 327(7417):745-748.

55. Halvorsen PA, Kristiansen IS. Decisions on drug therapies by numbers needed to treat: a randomized trial. Arch Intern Med. 2005;165(10): $1140-1146$.

56. Goodyear-Smith F, Arroll B, Chan L, Jackson R, Wells S, Kenealy T. Patients prefer pictures to numbers to express cardiovascular benefit from treatment. Ann Fam Med. 2008;6(3):213-217. 
57. Goldman RE, Parker DR, Eaton CB, et al. Patients' perceptions of cholesterol, cardiovascular disease risk, and risk communication strategies. Ann Fam Med. 2006;4(3):205-212.

58. Garcia-Retamero R, Galesic M. Who profits from visual aids: overcoming challenges in people's understanding of risks. Soc Sci Med. 2010; 70(7):1019-1025.

59. Tait AR, Voepel-Lewis T, Zikmund-Fisher BJ, Fagerlin A. The effect of format on parents' understanding of the risks and benefits of clinical research: a comparison between text, tables, and graphics. $J$ Health Commun. 2010;15(5):487-501.

60. Thomson RG, Eccles MP, Steen IN, et al. A patient decision aid to support shared decision-making on anti-thrombotic treatment of patients with atrial fibrillation: randomised controlled trial. Qual Saf Health Care. 2007;16(3):216-223.

61. Bickmore TW, Pfeifer LM, Byron D, et al. Usability of conversational agents by patients with inadequate health literacy: evidence from two clinical trials. J Health Commun. 2010;15(Suppl 2):197-210.

62. Protheroe J, Fahey T, Montgomery AA, Peters TJ. Effects of patients' preferences on the treatment of atrial fibrillation: observational study of patient-based decision analysis. BMJ. 2000;320(7246):1380-1384.

63. Lenert LA, Ziegler J, Lee T, Unfred C, Mahmoud R. The risks of multimedia methods: effects of actor's race and gender on preferences for health states. J Am Med Inform Assoc. 2000;7(2):177-185.

64. O'Connor AM, Bennett CL, Stacey D, et al. Decision aids for people facing health treatment or screening decisions. Cochrane Database Syst Rev. 2009;8(3):CD001431.

65. Ellis DA, Hopkin JM, Leitch AG, Crofton J. "Doctors' orders": controlled trial of supplementary, written information for patients. BMJ. 1979;1(6161):456.

66. USA Department of Health and Human Services. Healthy people 2010: understanding and improving health. 2nd ed. Washington, DC: US Government Printing Office; 2000.

67. Kutner M, Greenberg E, Jin Y, Paulsen C; for the National Center for Education Statistics. The health literacy of America's adults: results from the 2003 National Assessment of Adult Literacy. (NCES 2006-483) Washington, DC: US Department of Education; 2006.

68. Australian Bureau of Statistics. Health literacy, Australia. Cat nr 4233.0. Canberra, Australia: Australian Bureau of Statistics; 2006.
69. Wolf MS, Williams MV, Parker RM, Parikh NS, Nowlan AW, Baker DW. Patients' shame and attitudes toward discussing the results of literacy screening. J Health Commun. 2007;12(8):721-732.

70. Committee on Health Literacy and Board on Neuroscience and Behavioral Health, Institute of Medicine. Health literacy: a prescription to end confusion. Washington, DC: The National Academy Press; 2004.

71. Halvorsen PA, Kristiansen IS, Aasland OG, Førde OH. Medical doctors' perception of the "number needed to treat" (NNT.) A survey of doctors' recommendations for two therapies with different NNT. Scand J Prim Health Care. 2003;21(3):162-166.

72. Rakow T, Bull C. Same patient, different advice: a study into why doctors vary. Arch Dis Child. 2003;88(6):497-502.

73. Montori VM, Leung TW, Devereaux PJ, et al. Can contraindications compromise evidence-based, patient-centered clinical practice? Can J Clin Pharmacol. 2006;13(1):e92-e101.

74. Street RL Jr, Gordon H, Haidet P. Physicians' communication and perceptions of patients: is it how they look, how they talk, or is it just the doctor? Soc Sci Med. 2007;65(3):586-598.

75. Beck RS, Daughtridge R, Sloane PD. Physician-patient communication in the primary care office: a systematic review. J Am Board Fam Pract. 2002;15(1):25-38.

76. Beach MC, Roter DL, Wang NY, Duggan PS, Cooper LA. Are physicians' attitudes of respect accurately perceived by patients and associated with more positive communication behaviors? Patient Educ Couns. 2006;62(3):347-354.

77. Hou J, Shim M. The role of provider-patient communication and trust in online sources in Internet use for health-related activities. $J$ Health Commun. 2010;15(Suppl 3):186-199.

78. Ruelaz AR, Diefenbach P, Simon B, Lanto A, Arterburn D, Shekelle PG. Perceived barriers to weight management in primary care-perspectives of patients and providers. J Gen Intern Med. 2007;22(4):518-522.

79. Westby MD, Backman CL. Patient and health professional views on rehabilitation practices and outcomes following total hip and knee arthroplasty for osteoarthritis: a focus group study. BMC Health Serv Res. 2010;10:119.

80. Street RL Jr, Haidet P. How well do doctors know their patients? Factors affecting physician understanding of patients' health beliefs. J Gen Intern Med. 2011;26(1):21-27.
Patient Intelligence

\section{Publish your work in this journal}

Patient Intelligence is an international, peer-reviewed, open access journal that characterizes and measures the central role of patient behavior and intention in optimizing healthcare management in all areas of disease and complaint types. An improved understanding of patient intelligence coupled with predictive analysis helps an organization contribute more effectively to achieving better outcomes.

\section{Dovepress}

The journal is characterized by the rapid reporting of reviews, original research, methodologies, analytics, modeling, clinical studies and patient surveys across all disease areas. The manuscript management system is completely online and includes a very quick and fair peer-review system. Visit http://www.dovepress.com/ testimonials.php to read real quotes from published authors. 